\title{
Comparison between Supraclavicular and Infraclavicular Brachial Plexus Block in Patients Undergoing Forearm Surgeries
}

\author{
Parvati Sreelal ${ }^{1}$, Amol Prakash Singam² ${ }^{2}$ Bhargav Vishnu Gantasala ${ }^{3}$ \\ ${ }^{1}$ Department of Anaesthesiology, Jawaharlal Nehru Medical College, Datta Meghe Institute of Medial \\ Sciences (DMIMS DU), Sawangi, Wardha, Maharashtra, India. ${ }^{2}$ Department of Anaesthesiology, \\ Jawaharlal Nehru Medical College, Datta Meghe Institute of Medial Sciences (DMIMS DU), Sawangi, \\ Wardha, Maharashtra, India. ${ }^{3}$ Department of Anaesthesiology, Jawaharlal Nehru Medical College, Datta \\ Meghe Institute of Medial Sciences (DMIMS DU), Sawangi, Wardha, Maharashtra, India.
}

\section{ABSTRACT}

\section{BACKGROUND}

Of the various modalities of achieving surgical anaesthesia of the forearm, brachial plexus block by injecting local anaesthetic is considered highly beneficial and practical. Supraclavicular and infra-clavicular approaches of brachial plexus blocks provide comprehensive anaesthesia for surgeries of the forearm. The primary outcome measured was the comparison of two blocks with respect to sparing of any dermatome, whereas the secondary outcomes measured were block performance time, duration of analgesia, and complications associated with each technique.

\section{METHODS}

Sixty adult patients of either sex belonging to the American Society of Anesthesiologists (ASA) physical status I and II in the age range of 20-70 years scheduled to undergo surgeries of the forearm were divided into two groups: Supraclavicular (SCB group) and Infraclavicular (ICB group) of 30 each. Both the blocks were given by $30 \mathrm{~mL}$ of $0.375 \%$ injection Bupivacaine using a $22 \mathrm{G}, 5 \mathrm{~cm}$ insulated needle and nerve locator. Both the groups were compared with respect to sparing of dermatomes, block performance time, duration of analgesia and complications like Horner's syndrome, vascular puncture, and pneumothorax. Statistical analysis was performed with Student unpaired t-test and Chi-square test and $p<0.05$ was considered to be statistically significant.

\section{RESULTS}

Block performance time was similar in both the groups. Duration of analgesia was comparable among the two groups. The incidence of incomplete radial block was significantly higher in ICB group as compared to SCB group $(p=0.046, S)$. Incidence of Horner's syndrome in SCB group were higher than in ICB group, but they were statistically insignificant. ( $p=0.15$, NS). One patient in SCB group had subclavian vein puncture as compared to none in ICB group and was statistically insignificant.

\section{CONCLUSIONS}

Supraclavicular approach for brachial plexus block provides reliable and comprehensive anaesthesia for forearm surgeries without any significant dermatomal sparing unlike infraclavicular approach. Both groups had similar block performance time and duration of analgesia for forearm surgeries. Even though SCB was associated with complications like Horner's syndrome and vascular puncture, it was transient and statistically insignificant. Hence supraclavicular approach is considered to be superior to infraclavicular approach.

\section{KEY WORDS}

Brachial Plexus Block, Infraclavicular Block, Supraclavicular Block, Nerve Locator, Forearm Surgery

\author{
Corresponding Author: \\ Dr. Amol Singam, \\ Professor and HOD, \\ Department of Anaesthesiology, \\ Jawaharlal Nehru Medical College, \\ Datta Meghe Institute of Medical Sciences, \\ Sawangi, Wardha-442001, Maharashtra, \\ India. \\ E-mail:dramolsingam@gmail.com
}

DOI: 10.14260/jemds/2020/416

Financial or Other Competing Interests: None.

How to Cite This Article:

Sreelal $P$, Singam AP, Gantasala BV. Comparison between supraclavicular and infraclavicular brachial plexus block in patients undergoing forearm surgeries. J. Evolution Med. Dent. Sci. 2020;9(26):19121915, DOI: $10.14260 /$ jemds/2020/416

Submission 17-04-2020,

Peer Review 02-06-2020,

Acceptance 09-06-2020,

Published 29-06-2020.

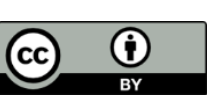




\section{BACKGROUND}

Of the various modalities of achieving surgical anaesthesia of the forearm, brachial plexus block by injecting local anaesthetic is considered highly beneficial and practical. Infraclavicular block is aimed at the brachial plexus in the infraclavicular space, where it is organized into three cords namely lateral, medial and posterior which surrounds the axillary artery. At this level, no major terminal branches arise.[1]

The infraclavicular block is advantageous due to innumerable reasons like it provides comprehensive anaesthesia of the upper limb by blocking the brachial plexus where the three cords most compactly run alongside the axillary artery. Injury to vital neurovascular structures in the neck and incidence of inadvertent pleural puncture is lower than with the interscalene and supraclavicular approaches. Infraclavicular block can be performed with the patient comfortably positioned as it does not require abduction of the arm at the shoulder.[2] However, there are case reports stating radial nerve sparing. ${ }^{[3]}$ Hence this study was undertaken to underline the incidence of radial nerve dermatomal sparing in infraclavicular block.

One of the several techniques used to anesthetize the brachial plexus is the supraclavicular block. It is an excellent choice for elbow and hand surgery as it provides anesthesia and analgesia to the upper extremity below the shoulder. The supraclavicular approach for brachial plexus block is often termed "spinal anaesthesia of the upper extremity" as it is a safe technique, with rapid onset and provides reliable anaesthesia for upper limb surgeries. The level at which this block is performed is at the level of the brachial plexus trunks, at this point almost the entire sensory, motor, and sympathetic innervation of the upper extremity is carried in just three nerve structures confined to a very small surface area. This approach technique typically provides a predictable, dense block with rapid onset. [3,4]

In our study we used nerve stimulation technique for brachial plexus block because of its high success rate and absence of complications which leads this to be a safe and effective technique than anatomical landmark guided technique. ${ }^{[5]}$

This prospective, randomized comparative study aimed to compare both the approach for brachial plexus block in patients posted for surgeries of the forearm. The primary outcome measured was the comparison of two blocks with respect to sparing of any dermatome, whereas the secondary outcomes measured were block performance time, duration of analgesia, and complications associated with each technique.

\section{METHODS}

After obtaining the Institutional Medical Ethics Committee approval and written informed consent, this prospective, randomized, comparative study was carried out in the Department of Anaesthesiology AVBRH affiliated to JNMC Sawangi (Meghe), Wardha, Maharashtra, India, during the period between April 2018 to April 2019.

Based on previous study by Koscielnaik-Nielsen et al,[6] infraclavicular block performance time was assumed to be 300 seconds. Keeping a study power $80 \%$ and alpha level of 0.05 , minimum 28 patients would be required in each group to for detection of $20 \%$ difference in block time. A sample size of 60 patients, with 30 patients in each group was taken to compensate for possible dropouts.

60 patients belonging to the American Society of Anesthesiologists (ASA) physical status I and II in the age range of 20-70 years scheduled to undergo surgeries of the forearm either elective or emergency were included in the study. The patients were counselled about the procedure, the advantages of postoperative pain relief with these techniques and their queries answered. Patients with known hypersensitivity to the study drug, patients on anticoagulant medications or with altered coagulation profile, obstetric population, patients with infection at the site of block and neck contracture or local bony deformity, patients with documented evidence of preoperative hemidiaphragmatic paresis, ptosis, and preoperative hoarseness of voice were excluded from the study.

All the patients selected for the study were kept nil per oral state of about 8 hours prior to the procedure. Local anaesthetic sensitivity testing was carried out using intradermal $0.1 \mathrm{~mL}$ of Injection Lignocaine 2\%. Intravenous access was obtained with 18G IV cannula. Preoperative sedation was deliberately avoided to minimize interference during the assessment of the quality of block and postoperative pain relief. The procedure was carried out in the operation theatre after ensuring a functional and working Boyle machine, suctioning equipment, intubation cart equipped with emergency drugs. Routine monitoring with Pulse Oximetry, electrocardiogram, noninvasive blood pressure was done and monitored throughout the procedure and baseline vital parameters noted.

The patients were randomly allocated into two equal groups of 30 each. Randomization was done by computer-generated random number table and allocation of the same was done in sealed envelope Technique. Opaque, sealed envelopes with the patient's registration number mentioned outside were handed over to an anaesthesiologist performing the block who was not taking part in the study. A paper slip inside them indicated either Infraclavicular block (ICB) group or supraclavicular block (SCB) group. After the patient arrived in the OT complex, he/she opened the envelope and administered either of the blocks to the patients.

There were two groups, namely ICB group and SCB group. Both the blocks were given with $30 \mathrm{~mL}$ of $0.375 \%$ injection Bupivacaine using a 22 G, $5 \mathrm{~cm}$ insulated needle. ${ }^{[7]}$ The nerve locator used was B Braun Stimulpex Dig Rc.

For the SCB group, the patients were asked to lie in supine position and after identifying the lateral border of the sternocleidomastoid, the fingers were slowly moved laterally to feel the interscalene groove between the anterior and medial scalene muscles. A point $1.5-2 \mathrm{~cm}$ above the clavicle was marked in the groove where the anterior scalene muscle could be palpated; pulsation of the subclavian artery was felt; and the Stimuplex needle was inserted in a backward, slightly medial, and posterior direction. Flexion and extension movement at the wrist at $0.4 \mathrm{~mA}$ was taken as the endpoint, and $30 \mathrm{~mL}$ of $0.375 \%$ Bupivacaine was injected using a $22 \mathrm{G}, 5$ $\mathrm{cm}$ insulated needle.

In patients belonging to ICB group, infraclavicular block of brachial plexus was performed using lateral coracoid approach. All aseptic precautions were taken, and coracoid 
process was identified. At a point about $2 \mathrm{~cm}$ medial and $2 \mathrm{~cm}$ inferior to coracoid process, an insulated stimulating needle was inserted perpendicular to the skin. The infraclavicular block was given using a nerve stimulator with a $22 \mathrm{G}$ insulated stimulator needle until the distal motor response like contraction of the middle and ring finger was elicited with a current of $0.5 \mathrm{~mA}$. $30 \mathrm{~mL}$ of $0.375 \%$ Bupivacaine was injected.

Ten minutes after giving the block sensory block, assessment was done over four major nerve distribution area (radial, ulnar, median, and musculocutaneous) on a three-point scale (0-normal sensation; 1-analgesia, blunt sensation; and 2-anaesthesia, no sensation). Motor blockade was evaluated by rating the muscle contraction forces corresponding to all four main branches of brachial plexus (ulnar, radial, median and musculocutaneous nerves) were located based on the specific twitches elicited by stimulation. Musculocutaneous nerve was identified by arm flexion. Radial nerve was isolated by arm and finger extension and supination while pronation and flexion of wrist, second and third finger identifies Median nerve. Flexion of fourth and fifth fingers and adduction of thumb identifies ulnar nerve. On a scale of $0-6$ where 6 denotes normal muscle force, 5 signifies slightly reduced muscle force, 4 corresponds to greatly reduced muscle force, 3 denotes slightly impaired mobility, 2 signifies greatly impaired mobility, 1 corresponds near complete paralysis, and 0 indicates complete paralysis, ${ }^{[8]}$ the block was assessed, and the surgery was allowed to proceed.

If 30 min after giving the block, the sensory and motor block were inadequate, then the block was considered to have failed and had to be supplemented with general anaesthesia. Block performance time was defined as the time interval from sterile skin preparation to the termination of injection and removal of nerve stimulator needle. Intraoperative complaints of pain, in case of radial nerve sparing was dealt by giving injection fentanyl $2 \mu \mathrm{g} / \mathrm{Kg}$ IV. Duration of surgery was defined as the time interval between incision to closure of the skin. Ten minutes after giving the block, all the patients were evaluated for any complication related to the block. ${ }^{9}$

Horner's syndrome was checked by watching for ptosis, miosis, and anhidrosis; phrenic nerve palsy was assessed by visualising bilateral excursion of the chest wall; vascular puncture was confirmed if there was visible blood in the syringe or any localised hematoma at the site of the puncture and hoarseness of voice was checked by talking to the patient. ${ }^{[8]}$ The severity of postoperative pain was evaluated by Visual Analog Scale (VAS) (0-10), where 0 indicates no pain and 10 indicates worst possible pain [10]. When the VAS $>4$ Injection diclofenac sodium $1.5 \mathrm{mg} / \mathrm{Kg} \mathrm{I} / \mathrm{M}$ for rescue analgesia. The duration of analgesia was taken as time from the onset of sensory block till the patient required first rescue analgesic. Chest X-ray is taken 8 hours after successful block to rule out complications like pneumothorax.[11]

\section{Statistical Analysis}

All the data were entered into the Excel sheet. Quantitative data were expressed as mean \pm standard deviation. Qualitative data were expressed as percentage. Statistical analysis was performed by using the descriptive and inferential statistics using the Chi-square test and Student's unpaired t-test. Software used in the analysis was SPSS 22.0 version and Graph
Pad Prism 6.0 version, and $\mathrm{P}<0.05$ was considered to be statistically significant.

\section{RESULTS}

Table 1 shows demographic characters, patients were comparable with respect to Age, Gender, ASA physical status and duration of surgery. Table 2 shows study variables, Block performance time was similar in both the groups. Duration of analgesia was comparable among the two group. The incidence of incomplete radial block was significantly higher in ICB group as compared to SCB group $(\mathrm{p}=0.046, \mathrm{~S})$. Table 3 shows complications, as cervical sympathetic chain is close to the brachial plexus in the supraclavicular region, the incidence of Horner syndrome in SCB group were higher than in ICB group, but they were statistically insignificant. ( $p=0.15$, NS) No additional treatment was required taking into account the temporary nature of the syndrome. One patient in SCB group had subclavian vein puncture as compared to none in ICB group. Pneumothorax was not reported in our study.

\begin{tabular}{|cccc|}
\hline Variables & $\begin{array}{c}\text { SCB Group } \\
(\mathbf{n = 3 0 )}, \mathbf{n}(\%)\end{array}$ & $\begin{array}{c}\text { ICB Group } \\
(\mathbf{n = 3 0 )}, \mathbf{n}(\%)\end{array}$ & P-Value \\
Age (years) & $36.78 \pm 4.61$ & $34.10 \pm 6.84$ & $0.08(\mathrm{NS})$ \\
Duration of surgery (min) & $94.28 \pm 42.23$ & $98.17 \pm 38.23$ & $0.7098(\mathrm{NS})$ \\
Gender: male / female & $23 / 7$ & $22 / 8$ & $1.00(\mathrm{NS})$ \\
ASA class I or II & $20 / 10$ & $19 / 11$ & $1.00(\mathrm{NS})$ \\
\hline \multicolumn{4}{c}{ Table 1. Demographic Data } \\
\hline
\end{tabular}

\begin{tabular}{|cccc|}
\hline Variables & $\begin{array}{c}\text { SCB Group } \\
(\mathbf{n = 3 0 )}, \mathbf{n}(\%)\end{array}$ & $\begin{array}{c}\text { ICB Group } \\
(\mathbf{n = 3 0 )}, \mathbf{n}(\%)\end{array}$ & P-Value \\
Radial nerve sparing & $1 / 30(3.33 \%)$ & $6 / 30(20 \%)$ & $0.046(\mathrm{~S})$ \\
$\begin{array}{c}\text { Block performance } \\
\text { time(seconds) }\end{array}$ & $224.54 \pm 34.38$ & $226.82 \pm 37.21$ & $0.8296(\mathrm{NS})$ \\
Duration of analgesia (min) & $738.42 \pm 24.26$ & $741.52 \pm 27.47$ & $0.64(\mathrm{NS})$ \\
\hline Table 2. Radial Nerve Sparing, Block Performance Time and \\
Duration of Analgesia
\end{tabular}

\begin{tabular}{|ccc|}
\hline Variables & $\begin{array}{c}\text { SCB Group } \\
(\mathbf{n = 3 0 )}, \mathbf{n}(\%)\end{array}$ & $\begin{array}{c}\text { ICB Group } \\
(\mathbf{n}=\mathbf{3 0}), \mathbf{n}(\%)\end{array}$ \\
Horner's syndrome & $2(6.66 \%)$ & 0 \\
Vascular puncture & $1(3.33 \%)$ & 0 \\
Pneumothorax & 0 & 0 \\
\hline \multicolumn{3}{c}{ Table 3. Complications } \\
\hline
\end{tabular}

\section{DISCUSSION}

Chin et al[12] compared various approaches of the brachial plexus with infraclavicular approach in terms of safety and efficacy. They concluded that infraclavicular block provides efficient anaesthesia for forearm surgeries and is also simple to learn and perform. This is consistent with our findings in terms of safety and efficacy. J. Desroches et al[13] in their study concluded that infraclavicular approach to brachial plexus block has a good tolerance to arm tourniquet and produces extensive sensory blockade for comprehensive anaesthesia for surgeries of the forearm.

Study conducted by Stav et al[14] and Dhir et al[15] showed similar block performance time was in the supraclavicular and the Infraclavicular brachial plexus block groups. Our results show comparable block performance time of $224.54 \pm 34.38$ seconds in SCB group and $226.82 \pm 37.21$ seconds in ICB group and a p value of 0.82 which is statistically insignificant. On the contrary, the block performance time was relatively quicker in 
Infraclavicular group $(9.57 \pm 3.19 \mathrm{~min})$ than Supraclavicular group (11.53 $\pm 2.90 \mathrm{~min})(\mathrm{P}=0.015)$ according to Abhinaya et al.[16]

Significant radial nerve sparing was noted in ICB group $(\mathrm{p}=$ $0.046, S$ ) in our study. Incomplete radial block by ICB group is encountered with single injection technique. Single injection technique of ICB does not often sufficiently block the posterior cord located deeper from the point of the needle entry. Infraclavicular approach of Brachial plexus block by double or triple injection technique to obtain full circumferential spread around the axillary artery aimed at medial, lateral and posterior cords showed significantly less radial sparing as evidenced by studies Sandhu et al[1] and Ootaki et al.[17] Comparable duration of analgesia was observed in both SCB and ICB groups in our study similar to the findings of Yang et al.[18] Incidence of complications like Horner's syndrome were 2 cases in SCB group and none in ICB group while one patient in SCB group had subclavian vein puncture as compared to none in ICB group, statistically insignificant. This is in accordance with the study by Yang et al.[18]

\section{CONCLUSIONS}

Supraclavicular approach for brachial plexus block provides reliable and comprehensive anaesthesia for forearm surgeries without any significant dermatomal sparing unlike infraclavicular approach. Both groups had similar block performance, time and duration of analgesia, for forearm surgeries. Even though SCB was associated with complications like Horner's syndrome and vascular puncture, it was transient and statistically insignificant. Hence supraclavicular approach is considered to be superior over infraclavicular approach for brachial plexus block.

\section{Limitations}

We did not explore other modalities of performing brachial plexus block like ultrasound guided approach. Neither did we compare supraclavicular and infraclavicular approaches of brachial plexus block to others like interscalene and axillary approaches.

\section{REFERENCES}

[1] Sandhu NS, Capan LM. Ultrasound-guided infraclavicular brachial plexus block. Br J Anaesth 2002;89(2):254-9.

[2] Macfarlane A, Anderson K. Infraclavicular brachial plexus blocks. Continuing Education in Anaesthesia, Critical Care Pain 2009;9(5):139-43.

[3] Supraclavicular brachial plexus block, 4th International NYSORA. https://www.nysora.com/supraclavicularbrachial-plexus-block.

[4] Taware M, Singam A, Chaudhari A. Comparison of buprenorphine and tramadol as an adjuvant to bupivacaine in supraclavicular brachial plexus block. Indian J Anaesth Analg 2018;5(1):22-7.
[5] Franco CD, Vieira ZE. 1,001 subclavian perivascular brachial plexus blocks: success with a nerve stimulator. Reg Anesth Pain Med 2000;25(1):41-6.

[6] Koscielniak-Nielsen ZJ, Frederiksen BS, Rasmussen H, et al. A comparison of ultrasound-guided supraclavicular and infraclavicular blocks for upper extremity surgery. Acta Anaesthesiol Scand 2009;53(5):620-6.

[7] Modak S, Basantwani S. Comparative study of $0.5 \%$ ropivacaine and $0.5 \%$ bupivacaine for brachial plexus block by supraclavicular approach for upper limb surgeries. International Journal of Basic \& Clinical Pharmacology 2017;5(4):1205-9.

[8] Ayyavu C, Rajamani G, Devi K, et al. A comparative study of brachial plexus block using infraclavicular (coracoid) and axillary approaches in forearm surgery. International Journal of Contemporary Medical Research 2016;3(9):2507-10.

[9] Mak PH, Irwin MG, Ooi CG, et al. Incidence of diaphragmatic paralysis following supraclavicular brachial plexus block and its effect on pulmonary function. Anaesthesia 2001;56(4):352-6.

[10] Bodian CA, Freedman G, Hossain S, et al. The visual analog scale for pain: clinical significance in postoperative patients. Anesthesiology 2001;95(6):1356-61.

[11] Neuburger M, Landes H, Kaiser H. Pneumothorax in vertical infraclavicular block of the brachial plexus. Review of a rare complication. Anaesthesist 2000;49(10):901-4.

[12] Chin KJ, Alakkad H, Adhikary SD, et al. Infraclavicular brachial plexus block for regional anaesthesia of the lower arm. Cochrane Database Syst Rev 2013;(8):CD005487.

[13] Desroches J. The infraclavicular brachial plexus block by the coracoid approach is clinically effective: an observational study of 150 patients. Can J Anaesth 2003;50(3):253-7.

[14] Stav A, Reytman L, Stav MY, et al. Comparison of the supraclavicular, infraclavicular and axillary approaches for ultrasound-guided brachial plexus block for surgical anesthesia. Rambam Maimonides Med J 2016;7(2):e0013.

[15] Dhir S, Brown B, Mack P, et al. Infraclavicular and supraclavicular approaches to brachial plexus for ambulatory elbow surgery: a randomized controlled observer-blinded trial. J Clin Anesth 2018;48:67-72.

[16] Abhinaya RJ, Venkatraman R, Matheswaran P, et al. A randomised comparative evaluation of supraclavicular and infraclavicular approaches to brachial plexus block for upper limb surgeries using both ultrasound and nerve stimulator. Indian J Anaesth 2017;61(7):581-6.

[17] Ootaki C, Hayashi H, Amano M. Ultrasound-guided infraclavicular brachial plexus block: an alternative technique to anatomical landmark-guided approaches. Reg Anesth Pain Med 2000;25(6):600-4.

[18] Yang CW, Kwon HU, Cho CK, et al. A comparison of infraclavicular and supraclavicular approaches to the brachial plexus using neurostimulation. Korean J Anesthesiol 2010;58(3):260-6. 